\title{
An Efficient Dynamical Model of Reluctance Actuators with Flux Fringing and Magnetic Hysteresis
}

\author{
Eduardo Moya-Lasheras ${ }^{* a}$, Carlos Sagues ${ }^{\mathrm{a}}$, Sergio Llorente ${ }^{\mathrm{b}}$ \\ ${ }^{a}$ Departamento de Informatica e Ingenieria de Sistemas (DIIS) and \\ Instituto de Investigacion en Ingenieria de Aragon (I3A), \\ Universidad de Zaragoza, Zaragoza 50018, Spain, \\ (e-mail: emoya@unizar.es, csagues@unizar.es) \\ ${ }^{b}$ Research and Development Department, \\ BSH Home Appliances Group, 50016 Zaragoza, Spain, \\ (e-mail: sergio.llorente@bshg.com)
}

\begin{abstract}
This paper presents an efficient and accurate dynamical model of reluctance actuators, suitable for prediction and control applications. It is a hybrid lumped-parameter state-space model that takes into account the mechanical and electromagnetic dynamics, including eddy currents, flux fringing, magnetic hysteresis and saturation. Special emphasis is placed on the hysteresis model, which is based on the Jiles-Atherton theory. The novel parts of the model - the gap reluctance expression and the modified JilesAtherton hysteresis model—are identified, showing that the simulated results fit very well the experimental data. Furthermore, its potential application for control is exemplified with a feedback strategy, in which the design of the controller and observer are based on the proposed dynamical model.
\end{abstract}

Keywords: Actuators, Electromagnetic devices, Lumped-parameter systems, Nonlinear models, Parameter identification

\section{Introduction}

Electromagnetic actuators are a form of electromechanical devices where the positioning of their movable parts rely on magnetic forces. Depending on the type of magnetic force, they are classified as Lorentz or reluctance actuators. The main advantage of reluctance actuators over Lorentz actuators is that they can achieve larger forces with lighter cores and reduced heat losses [1]. Therefore, they are ideal for small low-cost electromagnetic actuators, such as on-off solenoid valves and electromechanical relays [2]. However, reluctance actuators are much more challenging to control than Lorentz actuators, because the reluctance force depends on both the magnetic flux and the actuator position in a highly nonlinear fashion. Furthermore, in the case of small low-cost actuators, the position sensing is impractical or much more expensive than the device itself. Therefore, dynamical modeling is essential for both controller and observer designing.

Concerning the electromagnetic modeling, there are two main approaches. On the one hand, finite element models (FEM) [3] allow to represent the dynamic behavior of these devices with great accuracy, but they are too computationally demanding for control applications. On the other hand, analytical lumped-parameter models are much less complex and thus may be suitable for real-time estimation and control. There is considerable literature concerning the representation of reluctance actuators with lumped-parameter models. However, most works neglect the effect of the magnetic hysteresis $[4,5,6]$. Some works do propose hysteresis models for actuators, but neglect the motion dynamics [7, 8, 9]. Recently, [2] proposed a model of the mechanical and electromagnetic dynamics, with special emphasis on the derived hysteresis solution based on the generalized Preisach model (GPM) to characterize the magnetic hysteresis. It is treated as the basis of the proposal of this paper.

The mayor drawback of the GPM is its computational complexity. It requires numerical integration in each time step, even if it is implemented in an efficient state-space form [10]. Thus, to design a dynamical model adequate for control applications, an alternative must be used. From a more general perspective, there are numerous hysteresis models in the literature for ferromagnetic materials, e.g. Jiles-Atherton model [11], play and stop models [12], or the generalized positivefeedback model [13]. The Jiles-Atherton (JA) model is one of the most widespread physics-inspired techniques. Regarding control applications, it has been used for hysteresis compensation of electromagnets [10]. However, to the best of the authors' knowledge, the JA theory has not yet been incorporated in a dynamical model of reluctance actuators.

In this paper, a hybrid lumped-parameter model is presented for characterizing both mechanical and electromagnetic dynamics of reluctance actuators. The main modeling contributions are the gap reluctance function-based on the flux fringing factor proposed by McLyman [14]—and, most notably, the magnetic field strength dynamic function-based on the inverse JA model [15] —, taking into account the magnetic hysteresis and saturation phenomena. Another highlight is the reduction of the model, which transforms the electromagnetic subsystem and ensures that its parameters are identifiable from measurements 


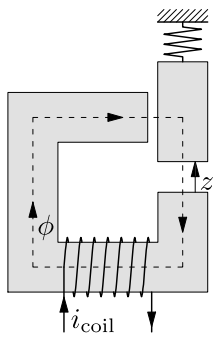

(a)

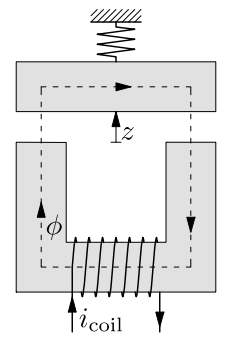

(b)

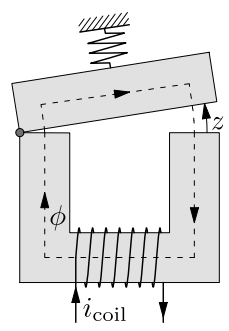

(c)
Figure 1: Schematic representation of single-coil reluctance actuators.

of electrical signals. Then, it is shown that the identified hysteresis model and gap reluctance expression adjust very well to experimental data with an accuracy similar to the state-of-theart actuator model, which is more computationally demanding. Additionally, to showcase the potential for control applications, an example of a feedback control is presented, in which both the controller and observer are designed based on the proposed model.

\section{Dynamical model}

\subsection{Reluctance actuators}

In Fig. 1, different reluctance actuators are represented as generalized schematics. For each one, the magnetic core is divided into two parts: a fixed part (stator) and a movable part (mover or armature). The air gap between them is dependent on the position of the mover, which is restricted between a lower and an upper limit. The position-and motion-can be linear (Figs. 1a and 1b) or angular (Fig. 1c). Low-cost actuators typically have a single coil and no permanent magnets. Consequently, the magnetic force has only one direction, attracting the mover toward the stator. To separate the mover from the stator, these devices rely on passive elastic forces.

In order to derive a computationally efficient lumpedparameter model, the complex distributed system must be approximated by a limited set of time-dependent scalar values, to which the state variables will be related. On the one hand, any movable part is assumed a rigid body, defined by its position $z$ and velocity $v$. On the other hand, the magnetic flux density $B$ and field strength $H$ are assumed to be constant for each core and gap section normal to the field lines. In this case, even if the area of the cross-section varies along the magnetic field path, the magnetic flux $\phi$ is uniform within it.

\subsection{Magnetic flux dynamics}

The electromagnetic subsystem is governed by two main equations. The first one is the electrical circuit equation of the coil,

$$
v_{\text {coil }}=R i_{\text {coil }}+N \dot{\phi},
$$

where $v_{\text {coil }}, i_{\text {coil }}, R$ and $N$ are the coil voltage, current, resistance and number of turns, respectively. The second equation is the Ampère's circuital law, which relates the total free current passing through a surface $\Sigma$ with the magnetic field strength $H$ across its closed boundary curve $\delta \Sigma$. Formally, it can be expressed as

$$
\iint_{\Sigma} J_{\text {free }} \mathrm{d} S=\oint_{\delta \Sigma} H \mathrm{~d} l,
$$

being $J_{\text {free }}$ the free current density. By defining $\delta \Sigma$ as the path of the magnetic flux (see Fig. 1), the left hand of (2) is

$$
\iint_{\Sigma} J_{\text {free }} \mathrm{d} S=N i_{\text {coil }}+i_{\text {eddy }}
$$

where $i_{\text {eddy }}$ is the net eddy current through the core. Under the assumption that the magnetic flux is uniform within the crosssection of the core, $i_{\text {eddy }}$ must be proportional to the magnetic flux derivative [2],

$$
i_{\text {eddy }}=-k_{\mathrm{e}} \dot{\phi}
$$

Regarding the right hand of (2), it is convenient to separate the integral into two components,

$$
\oint_{\delta \Sigma} H \mathrm{~d} l=\int_{\delta \Sigma_{\text {air }}} H \mathrm{~d} l+\int_{\delta \Sigma_{\text {core }}} H \mathrm{~d} l,
$$

because the model must take into account different magnetic phenomena in the air and the core.

The magnetic field intensity in the air gaps can be related to the magnetic flux through Hopkinson's law,

$$
\int_{\delta \Sigma_{\text {air }}} H \mathrm{~d} l=\mathcal{R}_{\mathrm{g}} \phi
$$

being $\mathcal{R}_{\mathrm{g}}$ the magnetic reluctance of the air gaps. It can be expressed in terms of the magnetic permeability of the air $\mu$, which is constant,

$$
\mathcal{R}_{\mathrm{g}}=\frac{\int H \mathrm{~d} l}{\phi}=\int \frac{\mathrm{d} l}{\mu A}, \quad \quad \mu=\frac{B}{H},
$$

where $A$ is the cross-sectional area. There can be several gaps of different lengths, so the integral can be interpreted as a sum of the different reluctance terms,

$$
\mathcal{R}_{\mathrm{g}}=\sum_{i} \frac{l_{\mathrm{g}_{i}}}{\mu A_{\mathrm{g}_{i}}}
$$

where the gap lengths $l_{\mathrm{g}_{i}}$ may depend on the mover position, and each effective area $A_{\mathrm{g}_{i}}$ augments with its corresponding gap length. This can be approximated using fringing flux factors for each gap, such as McLyman's [14],

$$
A_{\mathrm{g}_{i}}=A_{\mathrm{g} 0_{i}}\left(1+\frac{l_{\mathrm{g}_{i}}}{\sqrt{A_{\mathrm{g} 0_{i}}}} \ln \left(\frac{2 l_{\mathrm{w}}}{l_{\mathrm{g}_{i}}}\right)\right),
$$

where $A_{\mathrm{g} 0_{i}}$ is the effective area when the gap length tends to zero, and $l_{\mathrm{w}}$ is the winding length.

The expression is then simplified under the assumption that all position-dependent gaps are identical and proportional to the position, i.e. $l_{\mathrm{g}_{i}}=k_{\mathrm{g}} z$ (being $k_{\mathrm{g}}$ a positive constant). This is a limiting factor of the applicability of the model, but it is still 
useful to characterize a wide range of low-cost reluctance actuators. The assumption holds for most linear-travel reluctance actuators-e.g., Figs. 1a and 1b. Furthermore, it may still be a good approximation for short-stroke actuators with angular positioning-e.g., Fig. 1c-because the angles are very small $\left(l_{\mathrm{g}_{i}} \approx k_{\mathrm{g}} \sin (z) \approx k_{\mathrm{g}} z\right)$.

Then, their total reluctance is equal to

$$
\sum_{i=1}^{n_{\mathrm{g}}} \mathcal{R}_{\mathrm{g}, i}(z)=n_{\mathrm{g}} \frac{k_{\mathrm{g}} z}{\mu\left(1+\frac{k_{\mathrm{g}} z}{\sqrt{A_{\mathrm{g} 0}}} \ln \left(\frac{2 l_{\mathrm{w}}}{k_{\mathrm{g}}}\right)\right)},
$$

where $n_{\mathrm{g}}$ is the number of position-dependent gaps. On the other hand, there may be gaps that are not dependent on the mover position. In that case, their reluctance terms are grouped in the constant $\mathcal{R}_{\mathrm{g}, 0}$. Then, by combining all constants, $\mathcal{R}_{\mathrm{g}}$ can be expressed as

$$
\mathcal{R}_{\mathrm{g}}(z)=\mathcal{R}_{\mathrm{g}, 0}+\sum_{i=1}^{n_{\mathrm{g}}} \mathcal{R}_{\mathrm{g}, i}(z)=\mathcal{R}_{\mathrm{g}, 0}+\frac{\mathcal{R}_{\mathrm{g}, 0}^{\prime} z}{1+k_{1} z \ln \left(k_{2} / z\right)},
$$

where

$$
\mathcal{R}_{\mathrm{g}, 0}^{\prime}=n_{\mathrm{g}} k_{\mathrm{g}} / \mu, \quad k_{1}=k_{\mathrm{g}} / \sqrt{A_{\mathrm{g} 0}}, \quad k_{2}=2 l_{\mathrm{w}} / k_{\mathrm{g}} .
$$

The constants $\mathcal{R}_{\mathrm{g}, 0}, \mathcal{R}_{\mathrm{g}, 0}^{\prime}, k_{1}$ and $k_{2}$ are to be fitted through identification from experimental or FEM data.

The partial derivative of the gap reluctance, necessary for determining the magnetic force (see Section 2.4), can be easily derived as

$$
\mathcal{R}_{\mathrm{g}}^{\prime}(z)=\frac{\partial \mathcal{R}_{\mathrm{g}}(z)}{\partial z}=\frac{\mathcal{R}_{\mathrm{g}, 0}^{\prime}\left(1+k_{1} z\right)}{\left(1+k_{1} z \ln \left(k_{2} / z\right)\right)^{2}} .
$$

Note that (11) and (13) are indeterminate for $z=0$ so, in that case, $\mathcal{R}_{\mathrm{g}}$ and $\mathcal{R}_{\mathrm{g}}^{\prime}$ must be calculated in the limit from the right,

$$
\lim _{z \rightarrow 0^{+}} \mathcal{R}_{\mathrm{g}}(z)=\mathcal{R}_{\mathrm{g}, 0}, \quad \quad \lim _{z \rightarrow 0^{+}} \mathcal{R}_{\mathrm{g}}^{\prime}(z)=\mathcal{R}_{\mathrm{g}, 0}^{\prime} .
$$

Regarding the second integral of (5), the relation between $\phi$ and $H$ in the ferromagnetic core presents a hysteretic behavior. In order to account for this phenomenon, the reluctance approach is discarded and instead a hysteresis model is used. For now, the integral is simplified in terms of the magnetic field strength in the core $H_{\mathrm{c}}$ - whose dynamics will be modeled in Section 2.3-and its total length $l_{\mathrm{c}}$,

$$
\int_{\text {core }} H \mathrm{~d} l=H_{\mathrm{c}} l_{\mathrm{c}} .
$$

Finally, substituting (3), (4), (5), (6) into (3), and isolating $\dot{\phi}$, the following differential equation is derived,

$$
\dot{\phi}=-\frac{\mathcal{R}_{\mathrm{g}}(z) \phi+H_{\mathrm{c}} l_{\mathrm{c}}}{k_{\mathrm{e}}}+\frac{N}{k_{\mathrm{e}}} i_{\text {coil }},
$$

where $i_{\text {coil }}$ would be the input. However, it is more common to control the actuators with $v_{\text {coil }}$. Then, from (1) and (16),

$$
\dot{\phi}=-\frac{R\left(\mathcal{R}_{\mathrm{g}}(z) \phi+H_{\mathrm{c}} l_{\mathrm{c}}\right)}{N^{2}+R k_{\mathrm{e}}}+\frac{N}{N^{2}+R k_{\mathrm{e}}} v_{\text {coil }} \text {. }
$$

Regardless of the input choice, the dynamic equation has the same structure,

$$
\dot{\phi}=f_{\phi}\left(z, \phi, H_{\mathrm{c}}, u\right),
$$

where the input $u$ can be $v_{\text {coil }}$ or $i_{\text {coil }}$.

\subsection{Magnetic field strength dynamics}

A model must be used that takes into account the magnetic hysteresis and saturation phenomena. The JA model allows relating $H_{\mathrm{c}}$ and the magnetic flux density of the core $B_{\mathrm{c}}$. The original formulation [11] permits calculating $B_{\mathrm{c}}$ from $H_{\mathrm{c}}$. Specifically, an ordinary differential equation of the form

$$
\frac{\mathrm{d} M_{\mathrm{c}}}{\mathrm{d} H_{\mathrm{c}}}\left(M_{\mathrm{c}}, H_{\mathrm{c}}, \dot{H}_{\mathrm{c}}\right)= \begin{cases}f_{\mathrm{JA}}^{+}\left(M_{\mathrm{c}}, H_{\mathrm{c}}\right), & \text { if } \dot{H}_{\mathrm{c}} \geq 0 \\ f_{\mathrm{JA}}^{-}\left(M_{\mathrm{c}}, H_{\mathrm{c}}\right), & \text { if } \dot{H}_{\mathrm{c}}<0\end{cases}
$$

is solved to obtain the magnetization of the core $M_{\mathrm{c}}$, and then $B_{\mathrm{c}}$ is calculated from $M_{\mathrm{c}}$ and $H_{\mathrm{c}}$. However, in this case, it is more appropriate to determine $H_{\mathrm{c}}$ from $B_{\mathrm{c}}$. Thus, the inverse JA model [15] is used, in which an alternative differential equation is proposed, which can be expressed compactly as

$$
\frac{\mathrm{d} M_{\mathrm{c}}}{\mathrm{d} B_{\mathrm{c}}}\left(M_{\mathrm{c}}, B_{\mathrm{c}}, \dot{B}_{\mathrm{c}}\right)=\left\{\begin{array}{ll}
f_{\mathrm{iJA}}^{+}\left(M_{\mathrm{c}}, B_{\mathrm{c}}\right), & \text { if } \dot{B}_{\mathrm{c}} \geq 0 \\
f_{\mathrm{iJA}}^{-}\left(M_{\mathrm{c}}, B_{\mathrm{c}}\right), & \text { if } \dot{B}_{\mathrm{c}}<0
\end{array} .\right.
$$

The complete process can be summarized as follows:

First, the anhysteretic magnetization of the core $\left(M_{\mathrm{an}}\right)$ and its derivative are obtained by using the Langevin function,

$$
\begin{aligned}
M_{\mathrm{an}} & =M_{\mathrm{sat}}\left(\operatorname{coth}\left(B_{\mathrm{e}} / b\right)-b / B_{\mathrm{e}}\right), \\
\mathrm{d} M_{\mathrm{an}} / \mathrm{d} B_{\mathrm{e}} & =M_{\text {sat }} / b\left(1-\operatorname{coth}^{2}\left(B_{\mathrm{e}} / b\right)+\left(B_{\mathrm{e}} / b\right)^{2}\right),
\end{aligned}
$$

where $M_{\text {sat }}$ and $b$ are constants to be identified. The effective flux density $B_{\mathrm{e}}$ is defined as

$$
B_{\mathrm{e}}=\mu_{0}\left(H_{\mathrm{c}}+\alpha M_{\mathrm{c}}\right)
$$

where $\alpha$ is the domain coupling factor and $\mu_{0}$ is the vacuum permeability. It depends on both the core field strength $H_{\mathrm{c}}$ and magnetization $M_{\mathrm{c}}$, which can be derived from $H_{\mathrm{c}}$ and the magnetic flux density $B_{\mathrm{c}}$,

$$
M_{\mathrm{c}}=B_{\mathrm{c}} / \mu_{0}-H_{\mathrm{c}} .
$$

Inversely, $B_{\mathrm{c}}$ can be expressed in terms of $B_{\mathrm{e}}$ and $M_{\mathrm{c}}$ from (23) and (24),

$$
B_{\mathrm{c}}=B_{\mathrm{e}}+\mu_{0}(1-\alpha) M_{\mathrm{c}} .
$$

Secondly, the irreversible magnetization $M_{\text {irr }}$ is given by the following differential equation,

$$
\frac{\mathrm{d} M_{\text {irr }}}{\mathrm{d} B_{\mathrm{e}}}=\delta \frac{M_{\mathrm{an}}-M_{\text {irr }}}{\mu_{0} \kappa}, \quad \delta=\operatorname{sgn}\left(\dot{B}_{\mathrm{c}}\right),
$$

where $\kappa$ is the spinning factor.

Thirdly, the total magnetization $M_{\mathrm{c}}$ can be expressed as a weighted sum depending on the reversibility factor $c$,

$$
M_{\mathrm{c}}=(1-c) M_{\mathrm{irr}}+c M_{\mathrm{an}} .
$$


Then, the differential equation (20) is given by the following expression, respectively,

$$
\frac{\mathrm{d} M_{\mathrm{c}}}{\mathrm{d} B_{\mathrm{c}}}=\frac{\mathrm{d} M_{\mathrm{c}} / \mathrm{d} B_{\mathrm{e}}}{\mathrm{d} B_{\mathrm{c}} / \mathrm{d} B_{\mathrm{e}}}
$$

where $\mathrm{d} M_{\mathrm{c}} / \mathrm{d} B_{\mathrm{e}}$ and $\mathrm{d} B_{\mathrm{c}} / \mathrm{d} B_{\mathrm{e}}$ are obtained by deriving (27) and (25),

$$
\begin{aligned}
\mathrm{d} M_{\mathrm{c}} / \mathrm{d} B_{\mathrm{e}} & =(1-c) \mathrm{d} M_{\text {irr }} / \mathrm{d} B_{\mathrm{e}}+c \mathrm{~d} M_{\text {an }} / \mathrm{d} B_{\mathrm{e}}, \\
\mathrm{d} B_{\mathrm{c}} / \mathrm{d} B_{\mathrm{e}} & =1+\mu_{0}(1-\alpha) \mathrm{d} M_{\mathrm{c}} / \mathrm{d} B_{\mathrm{e}} .
\end{aligned}
$$

Note that, although (28) is a function of $B_{\mathrm{c}}, M_{\mathrm{c}}$ and the sign of $\dot{B}_{\mathrm{c}}$, the inverse differential permeability $\mathrm{d} H_{\mathrm{c}} / \mathrm{d} B_{\mathrm{c}}$ can be expressed as a function of $\phi, H_{\mathrm{c}}$ and $\dot{\phi}$,

$$
\frac{\mathrm{d} H_{\mathrm{c}}}{\mathrm{d} B_{\mathrm{c}}}\left(\phi, H_{\mathrm{c}}, \dot{\phi}\right)=\frac{1}{\mu_{0}}-\frac{\mathrm{d} M_{\mathrm{c}}}{\mathrm{d} B_{\mathrm{c}}}\left(B_{\mathrm{c}}, M_{\mathrm{c}}, \dot{B}_{\mathrm{c}}\right),
$$

where

$$
B_{\mathrm{c}}=\frac{\phi}{A_{\mathrm{c}}}, \quad M_{\mathrm{c}}=\frac{\phi}{\mu_{0} A_{\mathrm{c}}}-H_{\mathrm{c}}, \quad \dot{B}_{\mathrm{c}}=\frac{\dot{\phi}}{A_{\mathrm{c}}} .
$$

Finally, the dynamic equation of $H_{\mathrm{c}}$ is derived as

$$
\dot{H}_{\mathrm{c}}=f_{H_{\mathrm{c}}}\left(z, \phi, H_{\mathrm{c}}, u\right)=\frac{\mathrm{d} H_{\mathrm{c}}}{\mathrm{d} B_{\mathrm{c}}}\left(\phi, H_{\mathrm{c}}, \dot{\phi}\right) \frac{f_{\phi}\left(z, \phi, H_{\mathrm{c}}, u\right)}{A_{\mathrm{c}}} .
$$

\subsection{Complete model}

The mechanical dynamics remains to be defined. During motion, the acceleration of the mover is given by Newton's second law, with three forces,

$$
m a=F_{\mathrm{e}}(z)+F_{\mathrm{f}}(z, v)+F_{\mathrm{mag}}(z, \phi),
$$

where $z, v$ and $a$ are the position, velocity and acceleration of the armature; $F_{\mathrm{e}}, F_{\mathrm{f}}, F_{\text {mag }}$ are the elastic, friction and magnetic forces; $\phi$ is the magnetic flux; and $m$ is the moving mass. The only force that can be controlled-albeit indirectly-is $F_{\text {mag }}$, which depends on the derivative of the gap reluctance and the magnetic flux [4],

$$
F_{\text {mag }}=-\frac{1}{2} \mathcal{R}_{\mathrm{g}}^{\prime}(z) \phi^{2}
$$

The passive forces $F_{\mathrm{e}}$ and $F_{\mathrm{f}}$ are functions that vary from case to case. Nonetheless, for the sake of proposing a complete model, it is assumed that the elastic force is given by an ideal spring, with an spring elastic constant $k_{\mathrm{s}}$ and resting position $z_{\mathrm{s}}$,

$$
F_{\mathrm{e}}=k_{\mathrm{s}}\left(z_{\mathrm{s}}-z\right)
$$

The friction force is assumed viscous, depending on the coefficient $c_{\mathrm{f}}$,

$$
F_{\mathrm{f}}=c_{\mathrm{f}} v .
$$

In a state space representation both the position and velocity are state variables. In the case of motion, their dynamics are defined as

$$
\begin{aligned}
& \dot{z}=v, \\
& \dot{v}=f_{v}(z, v, \phi)=\frac{F_{\mathrm{e}}(z)+F_{\mathrm{f}}(z, v)+F_{\mathrm{mag}}(z, \phi)}{m} .
\end{aligned}
$$

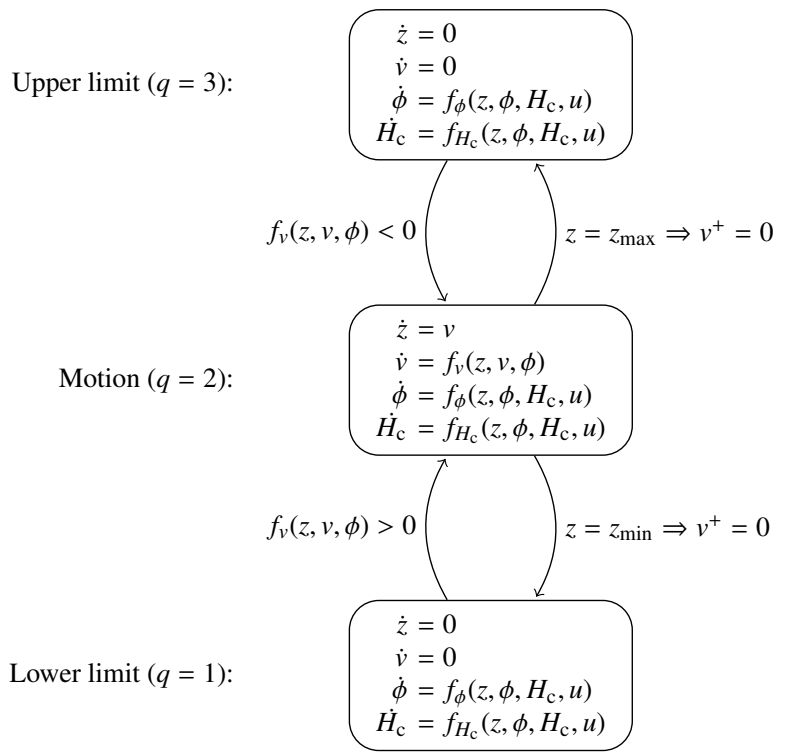

Figure 2: Diagram of the hybrid automaton that models the dynamics of reluctance actuators.

On the other hand, in the case that the mover has reached the lower limit $z_{\min }$ or the upper limit $z_{\max }$, it remains in that position until the unconstrained acceleration (34) is positive (if $z=z_{\min }$ ) or negative (if $z=z_{\max }$ ). Ultimately, the complete dynamical system can be represented through a hybrid automaton with three dynamic modes (lower limit, motion and upper limit), as shown in Fig. 2. Each transition is accompanied by its guard condition. Moreover, there are reset conditions $\left(v^{+}=0\right)$ in the case of transitioning to one of the limits. The dynamic equations can be expressed compactly as follows:

$$
\dot{\boldsymbol{x}}=\boldsymbol{f}_{q}(\boldsymbol{x}, u)
$$

where $\boldsymbol{x}=\left[\begin{array}{llll}z & v & \phi & H_{\mathrm{c}}\end{array}\right]^{\top}$ is the continuous state vector. Note that the state function $f_{q}$ depends also on the discrete state $q \in$ $\{1,2,3\}$.

Note that the magnetic flux $\phi$ may be considered the input of the mechanical system. However, its dynamics is dependent on the input of the electromagnetic system: $v_{\text {coil }}$ or $i_{\text {coil }}$. Once the input is selected, the other electrical variable-which is easily measured-can be considered the system output. If $v_{\text {coil }}$ is the input, the output $i_{\text {coil }}$ is calculated as

$$
i_{\text {coil }}=\frac{N\left(\mathcal{R}_{\mathrm{g}}(z) \phi+H_{\mathrm{c}} l_{\mathrm{c}}\right)}{N^{2}+R k_{\mathrm{e}}}+\frac{k_{\mathrm{e}}}{N^{2}+R k_{\mathrm{e}}} v_{\text {coil }} .
$$

If, instead, $i_{\text {coil }}$ is the input, the output $v_{\text {coil }}$ is

$$
v_{\text {coil }}=-\frac{N\left(\mathcal{R}_{\mathrm{g}}(z) \phi+H_{\mathrm{c}} l_{\mathrm{c}}\right)}{k_{\mathrm{e}}}+\frac{N^{2}+R k_{\mathrm{e}}}{k_{\mathrm{e}}} i_{\text {coil }} .
$$

In any case, the output equation can be expressed as

$$
y=h\left(z, \phi, H_{\mathrm{c}}, u\right) .
$$

where $u=i_{\text {coil }}$ and $y=v_{\text {coil }}$, or vice versa. 


\section{Model discussion}

In this section, the proposed dynamical model is discussed. First, some symmetry properties are presented. There is an even symmetric relation in the mechanical system,

$$
f_{v}(z, v, \phi)=f_{v}(z, v,-\phi)
$$

and an odd symmetric relation in the electromagnetic system,

$$
\begin{aligned}
f_{\phi}\left(z, \phi, H_{\mathrm{c}}, u\right) & =-f_{\phi}\left(z,-\phi,-H_{\mathrm{c}},-u\right), \\
f_{H_{\mathrm{c}}}\left(z, \phi, H_{\mathrm{c}}, u\right) & =-f_{H_{\mathrm{c}}}\left(z,-\phi,-H_{\mathrm{c}}-u\right) .
\end{aligned}
$$

Consequently, a position trajectory $z(t)$, given the initial conditions $z(0), v(0), \phi(0), H_{\mathrm{c}}(0)$ and an input signal $u(t)$ is equivalent to $z(t)$, given $z(0), v(0),-\phi(0),-H_{\mathrm{c}}(0)$ and $-u(t)$. In control applications, it should be borne in mind that, when $\phi>0$, the magnetic force increases if $\dot{\phi}>0$; and when $\phi<0$, the magnetic force increases if $\dot{\phi}<0$. Therefore, to simplify the control design, it is recommendable to constrain $\phi$ or $u$ to non-negative values.

Secondly, the equilibrium conditions are established. In general, a state vector is an equilibrium point $\boldsymbol{x}^{\mathbf{e q}}$ if

$$
\boldsymbol{f}_{q}\left(\boldsymbol{x}^{\mathrm{eq}}, u\right)=\mathbf{0} .
$$

For this particular system, there are multiple solutions for each input value $u$. In the case of motion, given an equilibrium position $z^{\mathrm{eq}}$, the equilibrium magnetic flux $\phi^{\mathrm{eq}}$ must be

$$
\phi^{\mathrm{eq}}= \pm \sqrt{2 \frac{F_{\mathrm{e}}\left(z^{\mathrm{eq}}\right)+F_{\mathrm{f}}\left(z^{\mathrm{eq}}, 0\right)}{\mathcal{R}_{\mathrm{g}}^{\prime}\left(z^{\mathrm{eq}}\right)}}, \quad \text { if } q=2
$$

On the other hand, if $z^{\mathrm{eq}}=z_{\min }(q=1)$ or $z^{\mathrm{eq}}=z_{\max }(q=3)$, there are infinite solutions that satisfy its corresponding inequation,

$$
\begin{array}{cc}
\left|\phi^{\mathrm{eq}}\right| \geq+\sqrt{2 \frac{F_{\mathrm{e}}\left(z_{\min }\right)+F_{\mathrm{f}}\left(z_{\min }, 0\right)}{\mathcal{R}_{\mathrm{g}}^{\prime}\left(z_{\min }\right)},} \quad \text { if } q=1, \\
\left|\phi^{\mathrm{eq}}\right| \leq+\sqrt{2 \frac{F_{\mathrm{e}}\left(z_{\max }\right)+F_{\mathrm{f}}\left(z_{\max }, 0\right)}{\mathcal{R}_{\mathrm{g}}^{\prime}\left(z_{\max }\right)}}, \quad \text { if } q=3 .
\end{array}
$$

Then, given $z^{\mathrm{eq}}$ and $\phi^{\mathrm{eq}}$, the equilibrium magnetic field strength $H_{\mathrm{c}}{ }^{\mathrm{eq}}$ is

$$
H_{\mathrm{c}}{ }^{\mathrm{eq}}=\frac{-\mathcal{R}_{\mathrm{g}}\left(z^{\mathrm{eq}}\right) \phi^{\mathrm{eq}}+N i_{\text {coil }}}{l_{\mathrm{c}}}, \quad v_{\text {coil }}=R i_{\text {coil }},
$$

which depends on the input ( $v_{\text {coil }}$ or $\left.i_{\text {coil }}\right)$. Therefore, each pair $\left(z^{\mathrm{eq}}, u\right)$ gives two equilibrium points $\boldsymbol{x}^{\mathrm{eq}}$ (in the case of motion), or infinite $\boldsymbol{x}^{\mathbf{e q}}$ (otherwise). Furthermore, the stability of these points can be studied by means of Lyapunov's indirect method, computing the Jacobian of $\boldsymbol{f}_{q}$ [16]. However, generalized analytical conditions are too complex, so the Jacobian should be computed numerically for each case.

Nonetheless, it is possible to represent the evolution of the electromagnetic variables in a generalized and intuitive way via

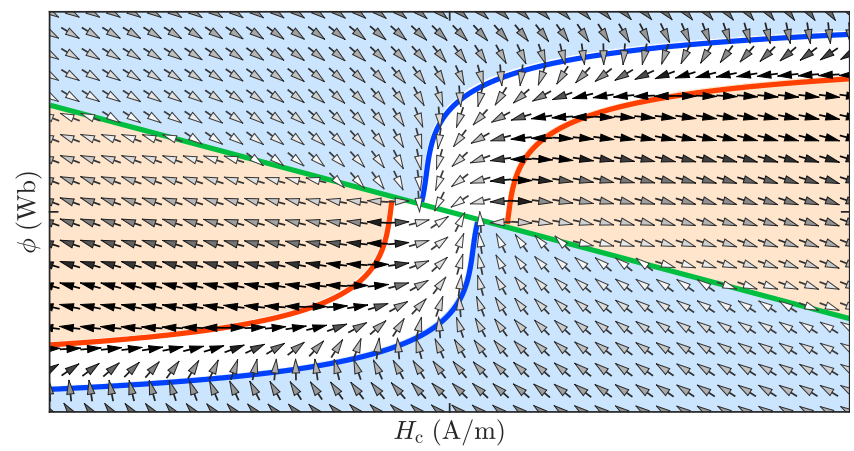

Figure 3: Vector field. Each point represents a vector $\left(H_{\mathrm{c}}, \phi\right)$, and each arrow represents its direction $\left(\dot{H}_{\mathrm{c}}, \dot{\phi}\right)$. Darker arrows indicate larger magnitudes.

a vector field, as in Fig. 3. The green line consists of the equilibrium points $\left(H_{\mathrm{c}}{ }^{\mathrm{eq}}, \phi^{\mathrm{eq}}\right)$ given by $(51)$. In general, the line is centered in $(0,0)$ if $u=0$, and it moves upwards as $u$ increases. Also, the slope of the line is steeper the larger $\mathcal{R}_{\mathrm{g}}(z)$, which increases with $z$. The blue lines are the points in which $\mathrm{d} H_{\mathrm{c}} / \mathrm{d} B_{\mathrm{c}}=0$ (see (31)). The red lines are the ones in which $\mathrm{d} B_{\mathrm{c}} / \mathrm{d} B_{\mathrm{e}}=0$ (see (30)), and they represent asymptotic discontinuities, in which $\left|\dot{H}_{\mathrm{c}}\right|$ tends to infinity.

The white region, between the red lines and the blue lines, is the physically feasible region, in which $\operatorname{sgn}(\dot{\phi})=\operatorname{sgn}\left(\dot{H}_{\mathrm{c}}\right)$, so the blue and red regions should be avoided. The blue region is not critical, because those points have derivatives that point toward the white region. The red region, however, may cause numerical errors, as their points diverge away from the white region. (During simulations, a red region may be reached, for example, with a large or variable time step and abrupt changes in $u$.) Nevertheless, it is easily remedied by modifying $\mathrm{d} B_{\mathrm{c}} / \mathrm{d} B_{\mathrm{e}}$ to

$$
\mathrm{d} B_{\mathrm{c}} / \mathrm{d} B_{\mathrm{e}}=\max \left(1+\mu_{0}(1-\alpha) \mathrm{d} M_{\mathrm{c}} / \mathrm{d} B_{\mathrm{e}}, \varepsilon\right),
$$

where $\varepsilon$ is a small positive constant. Thus, the points inside the red region would rapidly move toward the white region $\left(\left|\dot{H}_{\mathrm{c}}\right| \gg|\dot{\phi}|, \operatorname{sgn}\left(\dot{H}_{\mathrm{c}}\right)=\operatorname{sgn}(\dot{\phi})\right)$.

\section{Model reduction and identifiability}

Although the presented characterization of the magnetic hysteresis is simpler than other state-of-the-art alternatives, the complete model is much more complex than analogous lumpedparameter models that neglect this phenomenon. Specifically, the number of parameters is quite large. In order to fit the model to any device, the parameters must be estimated using data from different sources, e.g., measured electrical signals, FEM simulations, or direct measurements of parameters.

Ultimately, identification with only electrical signals is not possible, as the dynamical model is over-parameterized. However, by performing various manipulations to the dynamic equations, it is possible to derive an equivalent model that depends on a smaller set of parameters, i.e. a reduced model. 


\subsection{Reduced model}

In this equivalent model, $v_{\text {coil }}$ and $i_{\text {coil }}$, being the measurable electrical signals, must remain as input and output-or vice versa. In addition, $z$ and $v$ must remain as state variables because they are the variables of interest for control purposes. By contrast, $\phi$ and $H_{\mathrm{c}}$ may be replaced by auxiliary variables that help to simplify the dynamic equations.

Specifically, we propose to replace $\phi$ with the flux linkage $\lambda=N \phi$, and $H_{\mathrm{c}}$ with the auxiliary variable $M_{\mathrm{c}}^{*}=M_{\mathrm{c}} l_{\mathrm{c}} / N$. Then, the dynamic equation of $\lambda$ is derived from (16) or (17), depending on the input choice,

$$
\begin{aligned}
& \dot{\lambda}=f_{\lambda}^{*}\left(z, \lambda, M_{\mathrm{c}}^{*}, u\right)=-\frac{\mathcal{R}_{\mathrm{g}}^{*}(z) \lambda-M_{\mathrm{c}}^{*}}{k_{\mathrm{e}}^{*}}+\frac{1}{k_{\mathrm{e}}^{*}} i_{\text {coil }}, \\
& \dot{\lambda}=f_{\lambda}^{*}\left(z, \lambda, M_{\mathrm{c}}^{*}, u\right)=-\frac{R\left(\mathcal{R}_{\mathrm{g}}^{*}(z) \lambda-M_{\mathrm{c}}^{*}\right)}{1+R k_{\mathrm{e}}^{*}}+\frac{1}{1+R k_{\mathrm{e}}^{*}} v_{\text {coil }},
\end{aligned}
$$

where $u=i_{\text {coil }}$ or $u=v_{\text {coil }}$, respectively, and $k_{\mathrm{e}}^{*}$ is a new parameter, which is related to $k_{\mathrm{e}}$ and $N$ from the full model,

$$
k_{\mathrm{e}}^{*}=\frac{k_{\mathrm{e}}}{N^{2}} .
$$

Note also the dependence on $\mathcal{R}_{\mathrm{g}}^{*}$ which is a linear transformation of the gap reluctance, and can be expressed using the same structure as (11):

$$
\mathcal{R}_{\mathrm{g}}^{*}(z)=\mathcal{R}_{\mathrm{g}, 0}^{*}+\frac{\mathcal{R}_{\mathrm{g}, 0}^{\prime}{ }^{*} z}{1+k_{1} z \ln \left(k_{2} / z\right)},
$$

where

$$
\mathcal{R}_{\mathrm{g}, 0}^{*}=\frac{\mathcal{R}_{\mathrm{g}, 0}+l_{\mathrm{c}} /\left(\mu_{0} A_{\mathrm{c}}\right)}{N^{2}} \quad \mathcal{R}_{\mathrm{g}, 0}^{\prime *}=\frac{\mathcal{R}_{\mathrm{g}, 0}^{\prime}}{N^{2}} .
$$

Analogously, the equations (21)-(33) must be tweaked to obtain the dynamic equation of the auxiliary variable $M_{\mathrm{c}}^{*}$, resulting in the following function:

$$
\dot{M}_{\mathrm{c}}{ }^{*}=f_{M_{\mathrm{c}}}^{*}\left(z, \phi, H_{\mathrm{c}}, u\right)=\frac{\mathrm{d} M_{\mathrm{c}}^{*}}{\mathrm{~d} \lambda}\left(\lambda, M_{\mathrm{c}}^{*}, \dot{\lambda}\right) f_{\lambda}^{*}\left(z, \lambda, M_{\mathrm{c}}^{*}, u\right),
$$

where the intermediary steps are

$$
\begin{aligned}
\lambda_{\mathrm{e}} & =\lambda+\mu_{0}\left(1-\alpha^{*}\right) M_{\mathrm{c}}^{*}, \\
M_{\mathrm{an}}^{*} & =M_{\mathrm{sat}}^{*}\left(\operatorname{coth}\left(\lambda_{\mathrm{e}} / b^{*}\right)-b^{*} / \lambda_{\mathrm{e}}\right), \\
\mathrm{d} M_{\mathrm{an}}^{*} / \mathrm{d} \lambda_{\mathrm{e}} & =M_{\mathrm{sat}}^{*} / b^{*}\left(1-\operatorname{coth}^{2}\left(\lambda_{\mathrm{e}} / b^{*}\right)+\left(\lambda_{\mathrm{e}} / b^{*}\right)^{2}\right), \\
M_{\mathrm{irr}}^{*} & =\left(M_{\mathrm{c}}^{*}-c M_{\mathrm{an}}^{*}\right) /(1-c), \\
\mathrm{d} M_{\mathrm{irr}}^{*} / \mathrm{d} \lambda_{\mathrm{e}} & =\operatorname{sgn}(\dot{\lambda})\left(M_{\mathrm{an}}^{*}-M_{\mathrm{irr}}^{*}\right) /\left(\mu_{0} \kappa^{*}\right), \\
\mathrm{d} M_{\mathrm{c}}^{*} / \mathrm{d} \lambda_{\mathrm{e}} & =(1-c) \mathrm{d} M_{\mathrm{irr}}^{*} / \mathrm{d} \lambda_{\mathrm{e}}+c \mathrm{~d} M_{\mathrm{an}}^{*} / \mathrm{d} \lambda_{\mathrm{e}}, \\
\mathrm{d} \lambda / \mathrm{d} \lambda_{\mathrm{e}} & =\max \left(1+\mu_{0} \alpha^{*} \mathrm{~d} M_{\mathrm{c}}^{*} / \mathrm{d} \lambda_{\mathrm{e}}, \varepsilon\right), \\
\mathrm{d} M_{\mathrm{c}}^{*} / \mathrm{d} \lambda & =\left(\mathrm{d} M_{\mathrm{c}}^{*} / \mathrm{d} \lambda_{\mathrm{e}}\right) /\left(\mathrm{d} \lambda / \mathrm{d} \lambda_{\mathrm{e}}\right),
\end{aligned}
$$

which, in turn, depend on new auxiliary parameters,

$$
\begin{aligned}
M_{\mathrm{sat}}^{*} & =M_{\mathrm{sat}} l_{\mathrm{c}} / N, & b^{*} & =b N A_{\mathrm{c}}, \\
\kappa^{*} & =\kappa N A_{\mathrm{c}}, & & \alpha^{*}=(1-\alpha) N^{2} A_{\mathrm{c}} / l_{\mathrm{c}} .
\end{aligned}
$$

Note that these intermediary steps consist in the calculation of values and derivatives of the auxiliary time-dependent variables $\lambda_{\mathrm{e}}=B_{\mathrm{e}} N A_{\mathrm{c}}, M_{\text {an }}^{*}=M_{\text {an }} l_{\mathrm{c}} / N$ and $M_{\text {irr }}^{*}=M_{\text {irr }} l_{\mathrm{c}} / N$.

Regarding the mechanical part, the dynamic function of $v$ must be modified considering that $\phi$ and $\mathcal{R}_{\mathrm{g}}(z)$ have been replaced by $\lambda$ and $\mathcal{R}_{\mathrm{g}}^{*}(z)$, respectively. The derived expression is

$$
\dot{v}=f_{v}^{*}(z, v, \lambda)=\frac{F_{\mathrm{e}}(z)+F_{\mathrm{f}}(z, v)+F_{\mathrm{mag}}^{*}(z, \lambda)}{m},
$$

where

$$
F_{\text {mag }}^{*}(z, \lambda)=-\frac{1}{2} \mathcal{R}_{\mathrm{g}}^{* *}(z) \lambda^{2}, \quad \mathcal{R}_{\mathrm{g}}^{* *}(z)=\frac{\partial \mathcal{R}_{\mathrm{g}}^{*}(z)}{\partial z} .
$$

Lastly, the output function is obtained from (41) or (42), depending on the input choice,

$$
\begin{aligned}
& y=h^{*}\left(z, \lambda, M_{\mathrm{c}}^{*}, u\right)=-\frac{\mathcal{R}_{\mathrm{g}}^{*}(z) \lambda-M_{\mathrm{c}}^{*}}{k_{\mathrm{e}}^{*}}+\frac{1+R k_{\mathrm{e}}^{*}}{k_{\mathrm{e}}^{*}} i_{\text {coil }}, \\
& y=h^{*}\left(z, \lambda, M_{\mathrm{c}}^{*}, u\right)=\frac{\mathcal{R}_{\mathrm{g}}(z) \lambda-M_{\mathrm{c}}^{*}}{1+R k_{\mathrm{e}}^{*}}+\frac{k_{\mathrm{e}}^{*}}{1+R k_{\mathrm{e}}^{*}} v_{\text {coil }},
\end{aligned}
$$

where $u=i_{\text {coil }}$ and $y=i_{\text {coil }}$, or $u=v_{\text {coil }}$ and $y=i_{\text {coil }}$, respectively.

As a result of the above manipulations, the number of parameters in the electromagnetic system (53)-(60) and output equations (63), (64) has been reduced by three. Specifically, ten parameters from the complete model are replaced by seven auxiliary parameters (see (55), (57) and (60)).

\subsection{Identifiability analysis}

A local structural identifiability analysis is performed to demonstrate that the reduced model is identifiable with only electrical signals, and no further reductions are possible. The model is quite complex for this type of analysis, so it is convenient to separate it into the electromagnetic and mechanical subsystems. As the novel ideas of the proposal are in the electromagnetic subsystem, this analysis is focused on that part. Thus, the position is assumed to be fixed, effectively nullifying the mechanical subsystem.

For a local identifiability analysis, an observabilityidentifiability matrix is constructed. For a given instant, output derivatives are derived as functions of the variables, parameters, and input derivatives. Formally, the expression for each output derivative can be obtained recursively,

$$
\begin{aligned}
& y^{(i+1)}\left(z, \lambda, M_{\mathrm{c}}, u, \ldots, u^{(i+1)}\right)= \\
& \frac{\partial y^{(i)}}{\partial \lambda} f_{\lambda}^{*}(\cdot)+\frac{\partial y^{(i)}}{\partial M_{\mathrm{c}}^{*}} f_{M_{\mathrm{c}}}^{*}(\cdot)+\sum_{j=1}^{i} \frac{\partial y^{(i)}}{\partial u^{(j)}} u^{(j)},
\end{aligned}
$$

being $y^{(0)}=h^{*}(\cdot)$. Then, a vector of outputs is constructed,

$$
Y(\boldsymbol{\theta})=\left[\begin{array}{llll}
y^{(0)} & y^{(1)} & \cdots & y^{(9)}
\end{array}\right]^{\top},
$$

where $\boldsymbol{\theta}$ is the vector of unknown variables and parameters.

$$
\boldsymbol{\theta}=\left[\begin{array}{llllllllll}
\lambda & M_{\mathrm{c}}^{*} & R & k_{\mathrm{e}}^{*} & M_{\mathrm{sat}}^{*} & b^{*} & c & \kappa^{*} & \alpha^{*} & \mathcal{R}_{\mathrm{g}}^{*}
\end{array}\right]^{\top} .
$$


Note that, as the position is assumed to be fixed, the gap reluctance $\mathcal{R}_{\mathrm{g}}$ is considered here an unknown constant. Then the rank of a Jacobian of $Y$ (66) should be evaluated. Constructing and evaluating the rank of such a matrix is intractable. However, the particularities of the proposed model can be exploited to reduce the complexity of the problem.

The electromagnetic variables are $\lambda$ and $M_{\mathrm{c}}^{*}$. While $M_{\mathrm{c}}^{*}$ is a hidden variable, $\lambda$ can be assumed known because it can be directly estimated from the electrical signals, as explained in [5] and [17].

Then, it is easy to prove that the internal resistance of the coil $R$ is identifiable independently of the other parameters of the model. Assuming that $v_{\text {coil }}, i_{\text {coil }}, \dot{\lambda}$ are known, it can be directly calculated from (1). E.g., in a steady state, it is simply

$$
R=v_{\text {coil }} / i_{\text {coil }}, \quad \text { if } \dot{\lambda}=0 .
$$

Moreover, consider the following differential equation, derived from the output function $h^{*}$,

$$
\mathrm{d} y=\left(\frac{\partial h^{*}}{\partial \lambda} f_{\lambda}^{*}(\cdot)+\frac{\partial h^{*}}{\partial M_{\mathrm{c}}^{*}} f_{M_{c}}^{*}(\cdot)\right) \mathrm{d} t+\frac{\partial h^{*}}{\partial u} \mathrm{~d} u .
$$

Evidently, a step in the input results in a step in the output, dependent only on the second addend. This can be checked in the limit as $\mathrm{d} t$ tends to zero. Then, from (63) or (64), $R$ and $k_{\mathrm{e}}^{*}$ can be related to the ratio of steps in the voltage and current, regardless on the input choice. Specifically,

$$
\lim _{\mathrm{d} t \rightarrow 0} \frac{\mathrm{d} v_{\text {coil }}}{\mathrm{d} i_{\text {coil }}}=\frac{1+R k_{\mathrm{e}}^{*}}{k_{\mathrm{e}}^{*}} .
$$

Therefore, the value of $k_{\mathrm{e}}^{*}$ can be uniquely calculated from the electrical signals and $R$, which is also known.

As $R, k_{\mathrm{e}}$ and $\lambda$ are assumed known, the new parameter vector has seven elements,

$$
\boldsymbol{\theta}^{\prime}=\left[\begin{array}{lllllll}
M_{\mathrm{c}}^{*} & M_{\mathrm{sat}}^{*} & b^{*} & c & \kappa^{*} & \alpha^{*} & \mathcal{R}_{\mathrm{g}}^{*}
\end{array}\right]^{\top} .
$$

Still, the symbolic computation of seven consecutive derivatives is memory intensive. To further simplify the process, we can consider that there is one step in the input so that the the flux changes direction. Formally, it can be expressed as

$$
y_{ \pm}=h^{*}\left(z, \lambda, M_{\mathrm{c}}, u_{ \pm}\right), \quad \operatorname{sgn}\left(\dot{\lambda}_{-}\right) \neq \operatorname{sgn}\left(\dot{\lambda}_{+}\right),
$$

where $u_{ \pm}, y_{ \pm}$and $\lambda_{ \pm}$are the input, output and flux linkage derivative immediately before (-) and after (+) the step. This distinction is usually useless for identifiability analysis of dynamical models, as they do not add new information to determine the unknown parameters and variables. However, in this case, the dynamic behavior of $M_{\mathrm{c}}$ changes with the sign of the magnetic flux (26). Thus, it is possible to construct an output vector with smaller derivatives,

$$
Y_{ \pm}\left(\boldsymbol{\theta}^{\prime}\right)=\left[\begin{array}{lllllll}
y_{+}^{(0)} & y_{-}^{(1)} & y_{+}^{(1)} & y_{-}^{(2)} & y_{+}^{(2)} & y_{-}^{(3)} & y_{+}^{(3)}
\end{array}\right]^{\top} .
$$

Then the model is locally identifiable for $\boldsymbol{\theta}^{*}$ if the Jacobian $J$,

$$
J_{i j}\left(\boldsymbol{\theta}^{\prime}\right)=\frac{\partial\left(Y_{ \pm}\right)_{i}}{\partial \theta_{j}^{\prime}}
$$

Table 1: Known parameters.

\begin{tabular}{lllllll}
\hline Parameter & Value & & & & Parameter & Value \\
\cline { 1 - 4 }$N$ & 1200 & & & $m$ & $1.6 \times 10^{-3} \mathrm{~kg}$ \\
$k_{\mathrm{e}}$ & 1630 & $\Omega^{-1}$ & & $k_{\mathrm{S}}$ & 55 & $\mathrm{~N} / \mathrm{m}$ \\
$l_{\mathrm{c}}$ & 0.055 & $\mathrm{~m}$ & & $z_{\mathrm{s}}$ & 0.015 & $\mathrm{~m}$ \\
$A_{\mathrm{c}}$ & $1.26 \times 10^{-5} \mathrm{~m}^{2}$ & & $c_{\mathrm{f}}$ & 0 & $\mathrm{~N} \mathrm{~s} / \mathrm{m}$ \\
$z_{\min }$ & 0 & $\mathrm{~m}$ & & $z_{\max }$ & $9 \times 10^{-4}$ & $\mathrm{~m}$ \\
\hline
\end{tabular}

evaluated in $\boldsymbol{\theta}^{*}$, has full rank. Given the proposed simplifications, this can be checked easily using a computer algebra system. The symbolic matrix is indeed full-rank, so the reduced electromagnetic model is structurally locally identifiable. Furthermore, it is theoretically possible to derive the algebraic conditions of identifiability dependent on the input and its derivatives and the parameters themselves. However, the resulting expressions are too complex and unmanageable. Alternatively, for a specific case, values can be given to $u, \ldots, u^{(3)}$ and $\boldsymbol{\theta}^{*}$ in order to numerically check the rank of $\boldsymbol{J}$.

\section{Model fitting and comparison}

The presented model introduces two new ideas with respect to previous works: the gap reluctance approximation, and the characterization of the magnetic hysteresis. To show the adequacy of these proposals, they are identified using data from an actual reluctance actuator, and the results are evaluated with respect to a state-of-the-art alternative [2]. For this comparison, the common parameters of both models are assumed to be known (see Table 1), and only the parameters related to the gap reluctance and the magnetization curve are identified. In this case, identifying the full model is equivalent to identifying the reduced model because there are enough known parameters.

For convenience, the model parameters to be fitted correspond to the full model from Section 4. Note that the number of parameters to be fitted would be the same for the full and reduced model. Then, considering that the rest of parameters are already set, the full model is identifiable in the same way as the reduced model, which is proven in Section 4.2.

The device is a plunger-type solenoid valve, as shown in Fig. 4. It has a cylindrically symmetrical steel core, where the fixed part is the stator and the plunger is the mover. There are essentially two gaps between these parts: one below the plungerwhose length is equal to the plunger position-, and one around the plunger-whose length is constant. It has a single coil and a spring, generating magnetic and elastic forces in opposite directions. Note that the geometry of the valve, as inferred from Fig. 4b, is a revolutionized version of the schematic diagram from Fig. 1a, so their magnetic circuits are equivalent.

\subsection{Gap reluctance}

The gap reluctance and its derivative have been previously characterized for different positions from FEM simulations using the geometry presented in Fig. 4b [18]. In order to make use of these data in a dynamical model, the reluctance for any 


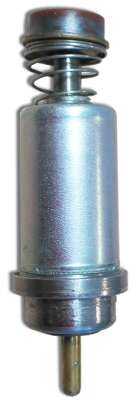

(a) Photo. (b) Longitudinal section.

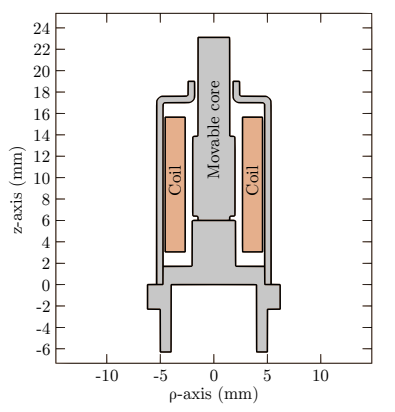

Figure 4: Solenoid valve.

Table 2: Estimated parameters.

\begin{tabular}{|c|c|c|c|}
\hline Parameter & Value & Parameter & Value \\
\hline $\mathcal{R}_{\mathrm{g}, 0}$ & $5.59 \times 10^{6} \quad \mathrm{H}^{-1}$ & $M_{\text {sat }}$ & $1.45 \times 10^{6} \mathrm{~A} / \mathrm{m}$ \\
\hline $\mathcal{R}_{\mathrm{g}, 0}^{\prime}$ & $1.11 \times 10^{11} \mathrm{H} / \mathrm{m}$ & $b$ & $2.45 \times 10^{-3} \mathrm{~T}$ \\
\hline$k_{1}$ & $1320 \quad \mathrm{~m}^{-1}$ & $c$ & 0.736 \\
\hline \multirow[t]{2}{*}{$k_{2}$} & $9.74 \times 10^{-3} \mathrm{~m}$ & $\kappa$ & $\mathrm{A} / \mathrm{m}$ \\
\hline & & $\alpha$ & $3.66 \times 10^{-3}$ \\
\hline
\end{tabular}

feasible position can be approximated through some type of interpolation. Alternatively, the proposed algebraic expression can be used. Thus, its parameters must be fitted.

Given the parameter vector,

$$
\boldsymbol{\theta}_{\mathbf{1}}=\left[\begin{array}{llll}
\mathcal{R}_{\mathrm{g}, 0} & \mathcal{R}_{\mathrm{g}, 0}^{\prime} & k_{1} & k_{2}
\end{array}\right]^{\top},
$$

the objective is to find the one that minimizes the errors of both $\mathcal{R}_{\mathrm{g}}$ and $\mathcal{R}_{\mathrm{g}}^{\prime}$. Formally, it is expressed as

$$
\min _{\theta_{1}}\left(\mathrm{NRMSE}_{\mathcal{R}_{\mathrm{g}}}{ }^{2}+\mathrm{NRMSE}_{\mathcal{R}_{\mathrm{g}}^{\prime}}{ }^{2}\right),
$$

where $\mathrm{NRMSE}_{\mathcal{R}_{\mathrm{g}}}$ is the normalized root-mean-square error of the simulated values $\mathcal{R}_{\mathrm{g}}{ }^{\text {sim }}$ with respect the experimental ones $\mathcal{R}_{\mathrm{g}}{ }^{\exp }$,

$$
\mathrm{NRMSE}_{\mathcal{R}_{\mathrm{g}}}=\sqrt{\frac{\sum_{i}\left(\mathcal{R}_{\mathrm{g}_{i}}^{\text {sim }}-\mathcal{R}_{\mathrm{g}_{i}}^{\text {exp }}\right)^{2}}{\sum_{i}\left(\mathcal{R}_{\mathrm{g}_{i}}^{\exp }\right)^{2}}},
$$

and $\mathrm{NRMSE}_{\mathcal{R}_{\mathrm{g}}^{\prime}}$ is defined in an equivalent manner. The optimized parameter are presented in the left half of Table 2. Fig. 5 shows that the algebraic expression with the fitted parameters matches very well the experimental data. The errors are

$$
\mathrm{NRMSE}_{\mathcal{R}_{\mathrm{g}}}=0.436 \%, \quad \mathrm{NRMSE}_{\mathcal{R}_{\mathrm{g}}^{\prime}}=0.226 \%
$$

\subsection{Magnetic hysteresis and saturation}

The most novel idea is the use of the inverse JA model for characterizing the magnetic hysteresis and saturation. To identify this part of the model, the hysteresis curve $i_{\text {coil }}-\phi$ is fitted using the same measurements as [2]. The experimental setup consists of a 4-quadrant power supplier (Toellner TOE 7621), a

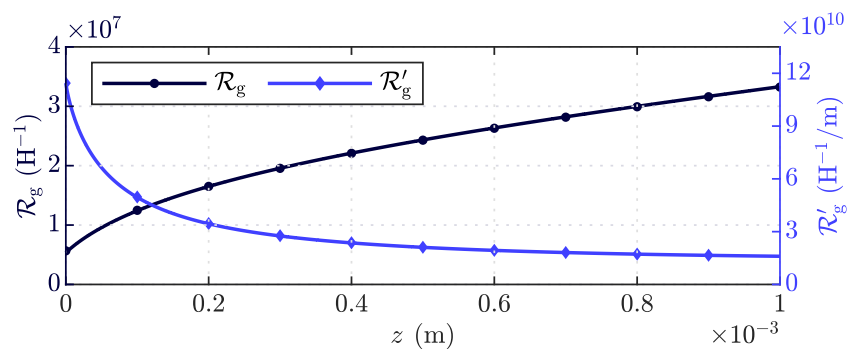

Figure 5: Gap reluctance and its derivative with respect to the gap length. The markers represent the experimental data and the lines represent the approximated results.

current probe (Tektronix TCP312A) with its corresponding amplifier (TCPA300), a USB oscilloscope with an arbitrary waveform generator (Picoscope 4824), a computer with MATLAB and the Instrument Control Toolbox installed, and the solenoid valve (Fig. 4). As only the electromagnetic subsystem is being fitted, the plunger position of the valve is fixed $\left(z=z_{\min }\right)$. Voltage signals are constructed in MATLAB, which are then sent out to the waveform generator of the USB oscilloscope. The generated signal is amplified by the power supplier, and applied to the solenoid valve. The applied signals are pulse waves with a frequency of $10 \mathrm{~Hz}$ and several amplitudes $(1 \mathrm{~V}, 2 \mathrm{~V}, 4 \mathrm{~V}, 6 \mathrm{~V}$, $9 \mathrm{~V})$. The applied voltage and current signals are measured with the oscilloscope at a sampling rate of $100 \mathrm{kHz}$ and sent to the computer. They are depicted in Fig. 6. Then, from those measurements, the magnetic flux is estimated following the method presented in [17].

Given the vector of parameters,

$$
\boldsymbol{\theta}_{\mathbf{2}}=\left[\begin{array}{lllll}
M_{\mathrm{sat}} & b & c & \kappa & \alpha
\end{array}\right]^{\top},
$$

the objective is to find the one that minimizes the errors of the simulated signals $\phi$ and $i_{\text {coil }}$, which are obtained by using the measured voltage signals as input $u$. Formally, the optimization problem is formulated as

$$
\min _{\theta_{2}}\left(\mathrm{wNRMSE}_{\phi,|\dot{\phi}|}^{2}+\mathrm{wNRMSE}_{i_{\text {coil }},|\dot{\phi}|}^{2}\right),
$$

where $\mathrm{wNRMSE}_{\phi,|\dot{ }|}$ is the weighted normalized root-meansquare error of $\phi$, with $|\dot{\phi}|$ acting as the weight,

$$
\operatorname{wNRMSE}_{\phi,|\dot{\phi}|}=\sqrt{\frac{\sum_{i}\left|\dot{\phi}_{i}^{\exp }\right|\left(\phi_{i}^{\text {sim }}-\phi_{i}^{\exp }\right)^{2}}{\sum_{i}\left|\dot{\phi}_{i}^{\exp }\right|\left(\phi_{i}^{\exp }\right)^{2}}},
$$

and ${ }^{W N R M S E} E_{i_{\text {coil }},|\dot{\phi}|}$ is defined in an equivalent manner. Note that $|\dot{\phi}|$ is used as the weight in order to avoid overfitting the slowly-varying intervals (specially the steady state intervals in which $\dot{\phi}=0$ ).

The optimization process results in the parameter values presented in the right half of Table 2. The simulated results fit fairly well the experimental data, as shown in Fig. 7. In particular, the average errors are

$$
{ }_{\mathrm{wNRMSE}},|\dot{\phi}|=1.956 \%, \quad \mathrm{wNRMSE}_{i_{\text {coil }}|\dot{\phi}|}=3.065 \% \text {. }
$$



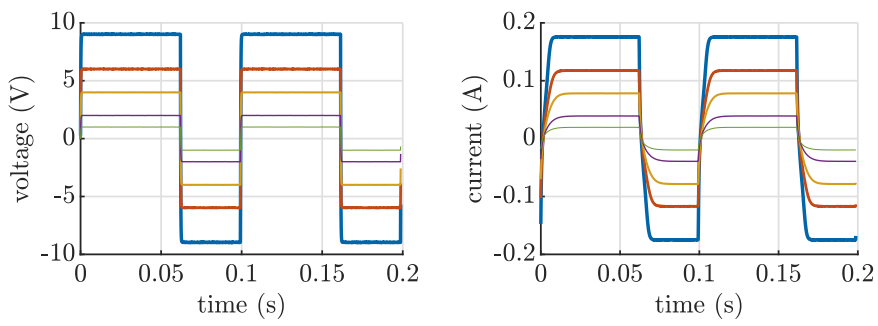

Figure 6: Measured voltage and current signals, used for model fitting.

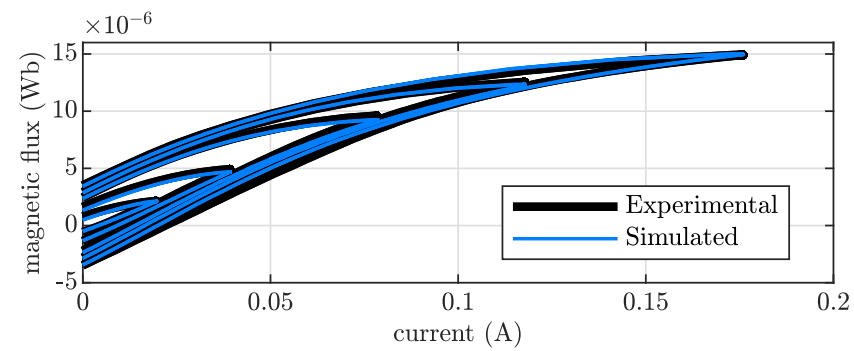

Figure 7: Experimental and simulated hysteresis curves. The part with negative currents is symmetric with respect to the origin.

For reference, the GPM from [2], which is much more computationally demanding, has also been fitted using the same procedure. Its average errors are

$\mathrm{wNRMSE}_{\phi,|\dot{\phi}|}=2.773 \%, \quad \mathrm{wNRMSE}_{i_{\text {coil }},|\dot{\phi}|}=2.688 \%$.

Consequently, there is no significant loss in accuracy.

\subsection{Model comparison}

The main advantage of the proposed model is its low computational requirements. To justify this statement, simulations are performed with the GPM-based and JA-based models. The latter also uses the reluctance gap approximation presented in Section 2.2, while the former directly interpolates from the experimental data (see Fig. 5). For this comparison, the parameters from (79) are readjusted to fit the GPM-based model, following the same procedure as in Section 5.2. Then, both models are simulated using as input a voltage signal with 3 pulses (see Fig. 8a). The resulting position and current are shown in Fig. $8 \mathrm{~b}$ and $8 \mathrm{c}$, respectively. Note that they match fairly well.

The simulations are performed using MATLAB's standard variable-step ordinary differential equation solver, ODE45, with default options; and a computer with a $2.4 \mathrm{GHz}$ Intel Core i7-5500 CPU. After 1000 repetitions, the mean computation time of the GPM-based model is $183.15 \mathrm{~ms}$. In contrast, the mean computation time of the JA-based model is $18.05 \mathrm{~ms}$, which is approximately ten times faster.

\section{Example of control application}

An important feature of the model is that it is suitable for control applications. To illustrate this, an example of a position

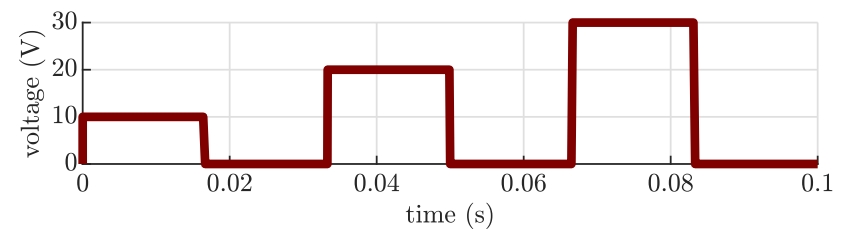

(a) Voltage (input).

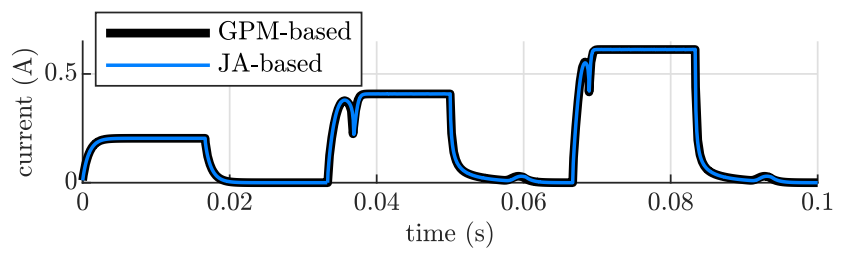

(b) Current (output).

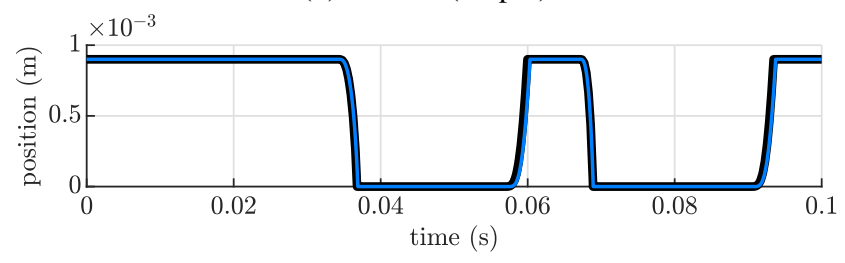

(c) Position (state variable).

Figure 8: Simulation results. The first voltage pulse is not sufficient to displace the mover.

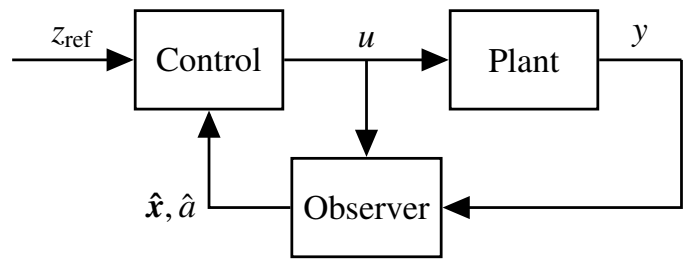

Figure 9: Control diagram.

feedback control is presented (see Fig. 9). Both controller and observer - which are based on state-of-the-art-techniques - are designed using the proposed model.

\subsection{Observer}

First, the observer is designed as a discrete-time unscented Kalman filter (UKF), using as basis the work presented in [5]. The structure of the observer is identical, except for the discretized model. In this case, the state vector is $\boldsymbol{x}^{k}=$ $\left[\begin{array}{llll}z^{k} & z^{k-1} & \phi^{k} & H_{\mathrm{c}}^{k}\end{array}\right]^{\top}$, and its state transition is

$$
\begin{aligned}
z^{k+1} & =\operatorname{sat}_{z_{\min }}^{z_{\max }}\left(2 z^{k}-z^{k-1}+T_{\mathrm{s}}^{2} f_{v}\left(z^{k}, v^{k}, \phi^{k}\right)+w_{z}^{k}\right), \\
z^{k} & =z^{k} \\
\phi^{k+1} & =\phi^{k}+T_{\mathrm{s}} f_{\phi}\left(z^{k}, \phi^{k}, H_{\mathrm{c}}^{k}, u^{k}\right)+w_{\phi}^{k}, \\
H_{\mathrm{c}}^{k+1} & =H_{\mathrm{c}}^{k}+T_{\mathrm{s}} f_{H_{\mathrm{c}}}\left(z^{k}, \phi^{k}, H_{\mathrm{c}}^{k}, u^{k}\right)+w_{H_{\mathrm{c}}}^{k},
\end{aligned}
$$

where the input $u^{k}$ may be the voltage or current; $T_{\mathrm{s}}$ is the sample time; $w_{z}^{k}, w_{\phi}^{k}$ and $w_{H_{\mathrm{c}}}^{k}$ account for the process noise (including discretization errors); and $\mathrm{sat}_{z_{\min }}^{z_{\max }}$ denotes a saturation function between $z_{\min }$ and $z_{\max }$. Moreover, $v^{k}$ is approximated as the 
previous average velocity,

$$
v^{k}=\frac{z^{k}-z^{k-1}}{T_{\mathrm{s}}},
$$

and the discrete state is directly estimated from $z^{k}$,

$$
q^{k}=\left\{\begin{array}{ll}
1, & \text { if } z^{k}=z_{\min } \\
3, & \text { if } z^{k}=z_{\max } \\
2, & \text { otherwise }
\end{array} .\right.
$$

The acceleration $a^{k}$ is also needed for the proposed controller. It can be derived from $\boldsymbol{x}^{k}$ and $q^{k}$,

$$
a^{k}=\left\{\begin{array}{ll}
f_{v}\left(z^{k}, v^{k}, \phi^{k}\right), & \text { if } q^{k}=2 \\
0, & \text { otherwise }
\end{array} .\right.
$$

\subsection{Controller}

Secondly, a controller is designed based on the theory of sliding-mode control [19], which has been already proposed for similar actuators [20]. The sliding surface is defined in terms of the position errors and their derivatives,

$$
\begin{aligned}
s & =\left(p+\frac{\mathrm{d}}{\mathrm{d} t}\right)^{2}\left(\hat{z}-z_{\text {ref }}\right) \\
& =\hat{a}-a_{\text {ref }}+2 p\left(\hat{v}-v_{\text {ref }}\right)+p^{2}\left(\hat{z}-z_{\text {ref }}\right),
\end{aligned}
$$

where $p$ is a positive constant to be adjusted; $z_{\text {ref }}, v_{\text {ref }}$ and $a_{\text {ref }}$ are the reference position, velocity and acceleration; and $\hat{z}, \hat{v}$ and $\hat{a}$ are the observed ones. Note that the variables are sampled as presented in Section 6.1, but, for clarity purposes, $k$ is removed from the expressions. In the case of motion, the action (voltage or current) is calculated as

$$
\begin{aligned}
u_{2}=-\frac{\frac{\partial f_{v}(\hat{\boldsymbol{x}})}{\partial \hat{\mathbf{z}}} \hat{v}+\frac{\partial f_{v}(\hat{\boldsymbol{x}})}{\partial \hat{v}} f_{v}(\hat{\boldsymbol{x}})+\frac{\partial f_{v}(\hat{\boldsymbol{x}})}{\partial \hat{\phi}} f_{\phi}(\hat{\boldsymbol{x}}, 0)}{\frac{\partial f_{v}(\hat{\boldsymbol{x}})}{\partial \hat{\phi}} \frac{\partial f_{\phi}(\hat{\boldsymbol{x}}, u)}{\partial u}} \\
+\frac{j_{\mathrm{ref}}-2 p\left(\hat{a}-a_{\mathrm{ref}}\right)-p^{2}\left(\hat{v}-v_{\mathrm{ref}}\right)-K s}{\frac{\partial f_{v}(\hat{\boldsymbol{x}})}{\partial f_{\phi}(\hat{\boldsymbol{x}}, u)}},
\end{aligned}
$$

where $\hat{\boldsymbol{x}}$ is the observed state vector, $\hat{\phi}$ is the observed magnetic flux, $j_{\text {ref }}=\dot{a}_{\text {ref }}$ is the reference jerk, and $K$ is a positive constant to be adjusted. Notice that, for clarity purposes, the dynamic functions have been shorten by using the state vector as an argument instead of the state variables, e.g., $f_{\phi}(\hat{\boldsymbol{x}}, u) \equiv f_{\phi}\left(\hat{z}, \hat{\phi}, \hat{H}_{\mathrm{c}}, u\right)$.

To prove that the system is asymptotically stable when applying $u_{2}$, a Lyapunov function is defined as $V=s^{2} / 2$. Its derivative is

$$
\dot{V}=s \dot{s}=s\left(\hat{j}-j_{\mathrm{ref}}+2 p\left(\hat{a}-a_{\mathrm{ref}}\right)+p^{2}\left(\hat{v}-v_{\mathrm{ref}}\right)\right),
$$

where $\hat{j}$ is the estimated jerk, which can be obtained from (39),

$$
\hat{j}=\frac{\mathrm{d} f_{v}(\hat{\boldsymbol{x}})}{\mathrm{d} t}=\frac{\partial f_{v}(\hat{\boldsymbol{x}})}{\partial \hat{z}} \hat{v}+\frac{\partial f_{v}(\hat{\boldsymbol{x}})}{\partial \hat{v} f_{v}(\hat{\boldsymbol{x}})+\frac{\partial f_{v}(\hat{\boldsymbol{x}})}{\partial \phi} f_{\phi}\left(\hat{\boldsymbol{x}}, u_{2}\right)} .
$$
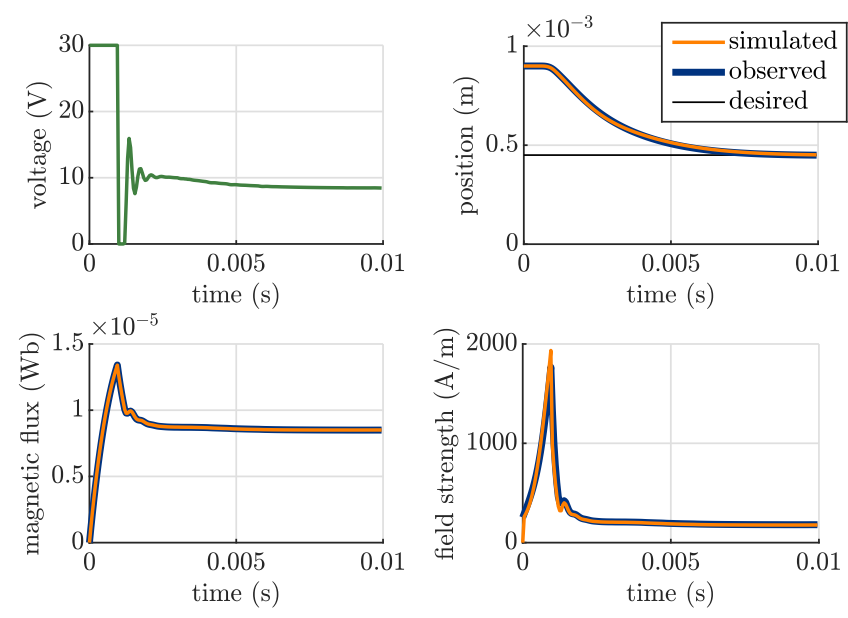

Figure 10: Control simulation.

Note that the dynamic equation of the magnetic flux is linear with respect to the action $u_{2}$, which means that it can be separated into two terms,

$$
f_{\phi}\left(\hat{\boldsymbol{x}}, u_{2}\right)=f_{\phi}(\hat{\boldsymbol{x}}, 0)+\frac{\partial f_{\phi}(\hat{\boldsymbol{x}}, u)}{\partial u} u_{2} .
$$

Then, by substituting (91), (92) and (89) into (90), the derivative of the Lyapunov function is simplified into

$$
\dot{V}=-K s^{2} .
$$

The Lyapunov function $V$ is lower bounded $(V \geq 0)$, and its derivative $\dot{V}$ is negative semi-definite $(\dot{V}=0$ if $s=0, \dot{V}<0$ otherwise). In that case, $V$ tends to 0 , which means that $s$ tends to 0 . Therefore, $\hat{z}$ tends to $z_{\text {ref }}$ (see (88)). Furthermore, in the case that the observed discrete state $\hat{q} \neq 2$, the minimum or maximum action is applied, depending on the position and the reference. In general, the action is

$$
u= \begin{cases}\operatorname{sat}_{0}^{u_{\max }}\left(u_{2}\right), & \text { if } \hat{q}=2 \\ u_{\max }, & \text { if } \hat{q}=1 \wedge z_{\mathrm{ref}}=z_{\min } \\ 0, & \text { if } \hat{q}=1 \wedge z_{\mathrm{ref}}>z_{\min } \\ 0, & \text { if } \hat{q}=3 \wedge z_{\mathrm{ref}}=z_{\max } \\ u_{\max }, & \text { if } \hat{q}=3 \wedge z_{\mathrm{ref}}<z_{\max }\end{cases}
$$

\subsection{Results}

The stability of the controller has been theoretically proved in the previous section. However, the sampling rate, input saturation and-more importantly-observation errors will negatively affect the control performance. Thus, as the last step, the control strategy is simulated to validate the combination of observer and controller, both based on the proposed model. The input or action is the voltage and the output is the current. The frequency of observer and controller is $20 \mathrm{kHz}$. To simulate the plant, the GPM-based model is used, in the same way as in Section 5.3. The desired position is set $z_{\text {ref }}=z_{\max } / 2$. For the plant simulation, the initial state is $z=z_{\max }, v=0, \phi=0$ and $H_{\mathrm{c}}=250 \mathrm{~A} / \mathrm{m}$. Regarding the observer, the initial guess 
is $\hat{\boldsymbol{x}}^{0}=\left[\begin{array}{llll}z_{\max } & z_{\max } & 0 & 0\end{array}\right]^{\top}$. Moreover the control parameters are set as $p=10^{3} \mathrm{~s}^{-1}$ and $K=10^{4} \mathrm{~s}^{-1}$. The results are presented in Fig. 10. The control strategy manages to maintain the position in the desired $z_{\text {ref }}$. During the transient response, there are some observer errors due to the discrepancies in the observer and plant model. However, the errors become practically negligible as the state variables reach a steady state.

\section{Conclusions}

In this paper we have presented a dynamical model of reluctance actuators, characterizing both mechanical and electromagnetic dynamics. It is a lumped-parameter model that takes into account the most relevant electromagnetic phenomena: hysteresis, saturation, flux fringing and eddy currents. It is a state-space representation, where every differential equation can be computed analytically. Thus, it can be used for designing observers or controllers with state-of-the-art techniques. The two main contributions are the gap reluctance expression and the hysteresis model.

On the one hand, the gap reluctance expression is derived using McLyman's factor in order to account flux fringing. The generalized expression can be directly used for actuators with an arbitrary number of position-dependent and fixed gaps. We have showed that it adjusts very well to experimental data from a specific device.

On the other hand, the magnetic hysteresis and saturation characterization is based on the Jiles-Atherton model. It is usually implemented in such a way that the input is the magnetization, and the output is the magnetic flux density. In the proposed modification, however, both the magnetic flux and field intensity are internal state variables, and the input and output are, interchangeably, the coil voltage and current. This approach makes the dynamical model directly applicable to control applications, in which the voltage can be controlled, and the current can be measured (or vice versa). It has been compared with a state-of-the-art solution that uses the Generalized Preisach Model, which requires to solve numerically the differential equation of the electromagnetic variable. Using experimental data from a specific device, it has been shown that the accuracy of both models are very similar, while the proposed model is much more computationally efficient.

\section{Acknowledgments}

This work was supported in part by the Ministerio de Ciencia Innovación y Universidades, Gobierno de España - European Union under project RTC-2017-5965-6 of subprogram RetosColaboración, in part by the Gobierno de Aragón-FSE 2014-20, in part by project DGA-T45_17R/FSE, and in part by project PGC2018-098719-B-I00 (MCIU/AEI/FEDER, UE).

\section{References}

[1] A. Katalenic, J. De Boeij, H. Butler, P. Van Den Bosch, Linearization of a current-driven reluctance actuator with hysteresis compensation, Mechatronics 23 (2) (2013) 163-171.
[2] E. Ramirez-Laboreo, M. G. L. Roes, C. Sagues, Hybrid Dynamical Model for Reluctance Actuators Including Saturation, Hysteresis and Eddy Currents, IEEE/ASME Trans. Mechatronics 24 (3) (2019) 1396-1406.

[3] R. R. Chladny, C. R. Koch, A. F. Lynch, Modeling automotive gasexchange solenoid valve actuators, IEEE Trans. Magn. 41 (3) (2005) 1155-1162.

[4] E. Ramirez-Laboreo, C. Sagues, S. Llorente, A New Model of Electromechanical Relays for Predicting the Motion and Electromagnetic Dynamics, IEEE Trans. Ind. Appl. 52 (3) (2016) 2545-2553.

[5] E. Moya-Lasheras, C. Sagues, E. Ramirez-Laboreo, S. Llorente, Nonlinear bounded state estimation for sensorless control of an electromagnetic device, in: 56th Annu. Conf. Decis. Control. CDC, IEEE, Melbourne, Australia, 2017, pp. 5050-5055.

[6] T. Braun, J. Reuter, J. Rudolph, Observer Design for Self-Sensing of Solenoid Actuators With Application to Soft Landing, IEEE Trans. Control Syst. Technol. 27 (4) (2019) 1720-1727.

[7] N. H. Vrijsen, J. W. Jansen, E. A. Lomonova, Prediction of Magnetic Hysteresis in the Force of a Prebiased E-Core Reluctance Actuator, IEEE Trans. Ind. Appl. 50 (4) (2014) 2476-2484.

[8] M. Ruderman, Stroke-dependent magnetic hysteresis modeling in proportional solenoids using parametric Gaussian-mixture Preisach distribution, in: IEEE/ASME Int. Conf. Adv. Intell. Mechatronics, AIM, Besançon, France, 2014, pp. 1587-1591.

[9] I. MacKenzie, D. Trumper, Real-time Hysteresis Modeling of a Reluctance Actuator Using a Sheared-Hysteresis-Model Observer, IEEE/ASME Trans. Mechatronics 21 (1) (2016) 4-16.

[10] S. Rosenbaum, M. Ruderman, T. Ströhla, T. Bertram, Use of JilesAtherton and Preisach Hysteresis Models for Inverse Feed-Forward Control, IEEE Trans. Magn. 46 (12) (2010) 3984-3989.

[11] D. C. Jiles, D. L. Atherton, Theory of ferromagnetic hysteresis, J. Magn. Magn. Mater. 61 (1-2) (1986) 48-60.

[12] S. Bobbio, G. Milano, C. Serpico, C. Visone, Models of magnetic hysteresis based on play and stop hysterons, IEEE Trans. Magn. 33 (6) (1997) 4417-4426.

[13] R. G. Harrison, Modeling High-Order Ferromagnetic Hysteretic Minor Loops and Spirals Using a Generalized Positive-Feedback Theory, IEEE Trans. Magn. 48 (3) (2012) 1115-1129.

[14] C. McLyman, Transformer and Inductor Design Handbook, Fourth Edition, CRC Press, 2011.

[15] N. Sadowski, N. Batistela, J. Bastos, M. Lajoie-Mazenc, An Inverse JilesAtherton Model to Take Into Account Hysteresis in Time-Stepping FiniteElement Calculations, IEEE Trans. Magn. 38 (2) (2002) 797-800.

[16] H. K. Khalil, Nonlinear Systems, Third Edition, Prentice Hall, Upper Saddle River, NJ, 2002.

[17] E. Ramirez-Laboreo, E. Moya-Lasheras, C. Sagues, Real-Time Electromagnetic Estimation for Reluctance Actuators, IEEE Trans. Ind. Electron. 66 (3) (2019) 1952-1961.

[18] E. Ramirez-Laboreo, C. Sagues, Reluctance actuator characterization via FEM simulations and experimental tests, Mechatronics 56 (2018) 58-66.

[19] J.-J. E. Slotine, W. Li, Applied Nonlinear Control, Vol. 199, Prentice Hall, Englewood Cliffs, NJ, 1991.

[20] P. Eyabi, G. Washington, Modeling and sensorless control of an electromagnetic valve actuator, Mechatronics 16 (3-4) (2006) 159-175. 